\title{
TU/e emonownen

\section{Robust vibration isolation by frequency-shaped sliding surface control with floor velocity/acceleration measurement}

\section{Citation for published version (APA):}

Ding, C., Damen, A. A. H., \& Bosch, van den, P. P. J. (2010). Robust vibration isolation by frequency-shaped sliding surface control with floor velocity/acceleration measurement. In Proceedings of the 36th Annual Conference of the IEEE Industrial Electronics Society, IECON 2010, 7-10 November 2010, Glendale, Arizona (pp. 1627-1632). Institute of Electrical and Electronics Engineers. https://doi.org/10.1109/IECON.2010.5675439

DOI:

10.1109/IECON.2010.5675439

Document status and date:

Published: 01/01/2010

\section{Document Version:}

Publisher's PDF, also known as Version of Record (includes final page, issue and volume numbers)

\section{Please check the document version of this publication:}

- A submitted manuscript is the version of the article upon submission and before peer-review. There can be important differences between the submitted version and the official published version of record. People interested in the research are advised to contact the author for the final version of the publication, or visit the $\mathrm{DOI}$ to the publisher's website.

- The final author version and the galley proof are versions of the publication after peer review.

- The final published version features the final layout of the paper including the volume, issue and page numbers.

Link to publication

\section{General rights}

Copyright and moral rights for the publications made accessible in the public portal are retained by the authors and/or other copyright owners and it is a condition of accessing publications that users recognise and abide by the legal requirements associated with these rights.

- Users may download and print one copy of any publication from the public portal for the purpose of private study or research.

- You may not further distribute the material or use it for any profit-making activity or commercial gain

- You may freely distribute the URL identifying the publication in the public portal.

If the publication is distributed under the terms of Article 25fa of the Dutch Copyright Act, indicated by the "Taverne" license above, please follow below link for the End User Agreement:

www.tue.nl/taverne

Take down policy

If you believe that this document breaches copyright please contact us at:

openaccess@tue.nl

providing details and we will investigate your claim. 


\title{
Robust Vibration Isolation by Frequency-Shaped Sliding Surface Control with Floor Velocity/Acceleration Measurement
}

\author{
C. Ding, A.A.H. Damen, P.P.J. van den Bosch
}

\begin{abstract}
This paper studies control of 1-DOF Active Vibration Isolation System (AVIS) using Frequency-Shaped Sliding Surface Control (FSSSC) approach based on the measurement schemes of the relative displacement and the floor absolute velocity/acceleration. Seismic velocity/acceleration sensors usually have large dimensions and mass which sometimes cause difficulties to fix them to the payload. The FSSSC approach has been recently applied to AVIS control and generalized as a twostep AVIS control design method. It is simple and has validated robust performance. Assuming linear regulation, both sliding surface design and the robustness of the realized performance are studied. The realized transmissibility is limited by the displacement sensor noise at high frequencies. The FSSSC designs of an example 1-DOF plant with the measurement schemes of the relative displacement and the floor absolute velocity/acceleration are provided. Theoretical calculations show that the robust low-frequency vibration isolation can be achieved but the performance robustness is limited at high frequencies due to the displacement sensor performance.
\end{abstract}

\section{INTRODUCTION}

Vibration isolation is essential for many high-precision applications, such as lithographic wafer steppers/scanners used to fabricate integrated circuits and electron microscopes used for sub-micron imaging [6]. The vibration source is typically from the floor. The Active Vibration Isolation System (AVIS) has been proved to be very effective to achieve low frequency vibration isolation and high capability of disturbance rejection. The vibration isolation performance is evaluated by the transmissibility, defined by the transfer function from the floor vibration to the payload vibration. The disturbance rejection performance is evaluated by the compliance, defined by the transfer function from the force disturbance to the payload vibration. Modal decomposition [4], [6] can be applied to simplify the multi-DOF control to 1 -DOF control. Therefore, a 1-DOF model can be used here as an example plant to study the AVIS control.

The objective of AVIS control is to minimize the plant absolute displacement (the terminology absolute indicates that this physical value is with respect to an inertially fixed reference). However, neither floor absolute displacement nor payload absolute displacement is directly measurable by any industrial sensors. Integration of absolute velocity/acceleration signal is not feasible because of the limited performance of the industrial sensors. The following two measurement schemes are widely implemented.

- The relative displacement (payload displacement with respect to the floor) and the payload absolute velocity.

- The relative displacement and the payload absolute acceleration.
However, seismic velocity/acceleration sensors which are suitable for low frequency measurement usually have large dimensions and mass. Sometimes it is difficult to fix these sensors on the payload especially when the payload is required to have limited dimensions and mass. In some contactless vibration isolation platforms, the power/signal wires connecting the sensor and the equipments on the floor induce extra disturbances. Therefore, AVIS control based on the following two measurement schemes are investigated.

- Relative displacement \& floor absolute velocity.

- Relative displacement \& floor absolute acceleration.

Each of them requires a Double-Input-Single-Output (DISO) controller for the 1-DOF AVIS control.

The well-known skyhook control [1] and the $H_{\infty}$ control [2], [3] are both applicable to this DISO controller design. However, the skyhook control is difficult to achieve the low-frequency vibration isolation due to the velocity sensor dynamics. The $H_{\infty}$ control is usually of high order and complicated. L. Zuo and J.J.E. Slotine [4] have applied the Frequency-Shaped Sliding Surface Control (FSSSC) to AVIS control assuming that ideal absolute velocity signal can be measured. The control structure is simple and easy to implement. Robust skyhook performance is validated by experiments. Previous study [7] generalizes the FSSSC approach to a two-step AVIS control design method for the measurement scheme of relative displacement and payload absolute velocity considering velocity sensor dynamics. The generalized FSSSC for the measurement scheme of relative displacement and payload absolute acceleration is also studied [5].

This paper studies the 1-DOF AVIS control using FSSSC approach based on the measurement schemes of the relative displacement and the floor absolute velocity/acceleration. The 1-DOF plant, sensor dynamics, and the AVIS performance requirements are introduced in Section II. The FSSSC design based on the two measurement schemes are described in Section III and Section IV. The example sliding-surfacestructures incorporating the sensor dynamics for the two measurement schemes are provided in Section V. The vibration isolation performance and its robustness of FSSSC based on the example 1-DOF plant are theoretically calculated in Section VI. This work is concluded in Section VII.

\section{Problem Statement}

\section{A. 1-DOF Model}

A 1-DOF model is introduced as an example plant to study the AVIS control. The physical model of the 1-DOF plant 
is shown in Fig. 1. The payload mass, spring stiffness, and damping coefficient are denoted by $m, k$, and $c$, respectively. For pneumatic suspension systems, $c>0$ and $k>0$. For 1DOF contactless electromagnetic isolators, $c=0$ and $k<0$ (For multi-DOF contactless electromagnetic isolators, there is at least one DOF that has negative stiffness). The payload absolute displacement, floor absolute velocity, floor absolute acceleration, and floor absolute displacement are denoted by $x_{A}, v_{G}, a_{G}$, and $x_{G}$, respectively. The actuator force is denoted by $f_{a}$. The equation of motion for the payload is given by

$$
m \ddot{x_{A}}+c \dot{x_{R}}+k x_{R}=f_{a},
$$

where $x_{R}=x_{A}-x_{G}$ is the relative displacement. The diagram of the physical model is shown in the dashed rectangular in Fig. 2. Define the transfer function $P$ as

$$
P(s)=\frac{1}{m s^{2}} \text {. }
$$

\section{B. Sensor Models}

The possible signals used for control are $x_{R}, v_{G}$ and $a_{G}$. The signals $\widetilde{x_{R}}, \widetilde{v_{G}}$, and $\widetilde{a_{G}}$ denote the measured $x_{R}, v_{G}$, and $a_{G}$, respectively. The displacement sensor usually have very high bandwidth (in the order of $10^{4} \mathrm{~Hz}$ ) so that the sensor dynamics is negligible at low frequencies (in the order of $10^{2}$ $\mathrm{Hz}$ or lower). The displacement sensor noise, denoted by $n_{x}$, is assumed to be independent of $x_{R}$ so that $\widetilde{x_{R}}$ is derived by

$$
\widetilde{x_{R}}=x_{R}+n_{x} \text {. }
$$

Geophone is a type of absolute velocity sensor widely used in the industry. The dynamic model [4], [8] for the geophone has the form of

$$
G_{v}(s)=\frac{s^{2}}{s^{2}+2 \omega_{v} \xi_{v} s+\omega_{v}^{2}},
$$

where $\omega_{v}$ is the resonant frequency and $\xi_{v}$ is the damping ratio. The geophone noise, denoted by $n_{v}$, is assumed to be independent of $v_{G}$. The relation between $\widetilde{v_{G}}$ and $v_{G}$ is

$$
\widetilde{v_{G}}=G_{v}(s) v_{G}+n_{v} \text {. }
$$

There are many types of acceleration sensors. The acceleration sensor based on piezoelectric effect is capable of acceleration measurement from zero frequency up to a certain resonant frequency $\omega_{a}$ (in the order of $10^{3}-10^{4} \mathrm{~Hz}$ ). At low frequencies, the sensor dynamics is negligible except there is usually a DC bias which can be classified as a part of the sensor noise. The acceleration sensor noise, denoted by $n_{a}$, is assumed to be independent of $a_{G}$. The relation between $\widetilde{a_{G}}$ and $a_{G}$ is

$$
\widetilde{a_{G}}=a_{G}+n_{a} .
$$

\section{Performance Requirements}

The closed-loop performances are the transmissibility $\mathbb{T}_{c}$, the compliance $\mathbb{C}_{c}$, the velocity/acceleration-sensor-noise sensitivity $\mathbb{S}_{c}$ and the displacement-sensor-noise sensitivity $\mathbb{R}_{c} . \mathbb{S}_{c}$ and $\mathbb{R}_{c}$ are concerned because they would affect $\left|\overline{\mathbb{T}_{c}}\right|$, the upper bound of $|\mathbb{T}| . \mathbb{S}_{c}$ is defined as the transfer function from the velocity/acceleration sensor noise to the payload absolute displacement and $\mathbb{R}_{c}$ is defined as the transfer

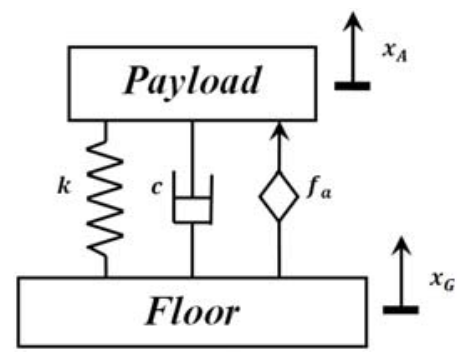

Fig. 1. Physical model of the 1-DOF plant.

function from the displacement sensor noise to the payload absolute displacement. The requirements are described as follows.

- $\mathbb{T}_{c}, \mathbb{C}_{c}, \mathbb{R}_{c}$, and $\mathbb{S}_{c}$ are all stable.

- Interested frequency range is from zero up to the order of $10^{2} \mathrm{~Hz}$.

- $\left|\mathbb{T}_{c}(\omega)\right|=0 \mathrm{~dB}, \forall \omega \in\left[0, \omega_{c}\right] .\left|\mathbb{T}_{c}(\omega)\right|$ decreases for all $\omega>\omega_{c}$ and the decreasing rate is at least $-40 \mathrm{~dB} / \mathrm{dec} . \omega_{c}$ is the cross-over frequency of $\mathbb{T}_{c}$. Smaller $\omega_{c}$ indicates better transmissibility. Around $\omega_{c},\left|\mathbb{T}_{c}\right|$ may have a peak. Smaller peak value indicates better transmissibility.

- Low $\left|\mathbb{C}_{c}(\omega)\right|$ for all $\omega \geqslant 0$. Some high performance AVIS require that $\left|\mathbb{C}_{c}(0)\right|=0(-\infty \mathrm{dB})$.

- $\left|\mathbb{S}_{\mathcal{c}}(0)\right|=0(-\infty \mathrm{dB})$. Low $\left|\mathbb{S}_{\mathcal{c}}(\omega)\right|$ for all $\omega>0$.

\section{FSSSC WITH FLOOR VELOCITY}

The application of "frequency-shaped sliding surface" to AVIS control was provided by L. Zuo and J.J.E. Slotine [4] in 2004. Therein, the sliding surface is designed for ideal feedback signals. The generalized FSSSC approach based on payload absolute acceleration and payload absolute velocity measurement are presented in [5] and [7], respectively. The linear FSSSC approach, including sliding surface design and the linear regulator design with floor absolute velocity measurement using geophone is studied in this section.

Fig. 2 shows the control diagram. The blocks in the dashed rectangular denotes the 1 -DOF plant. The sliding surface is defined by the equation $\sigma=0$. The blocks $\Lambda_{1}$ and $\Lambda_{2}$ are two transfer functions used to shape the sliding surface. The block $R$ is the regulator. The FSSSC design takes two steps. The first step is to design the frequencyshaped sliding surface $\left(\Lambda_{1}\right.$ and $\left.\Lambda_{2}\right)$ which determines the designed performances. The designed sliding surface and the original plant $P$ form a new system $P_{n}$, shown as the shaded blocks in Fig. 2. The second step is to design the regulator $R$ for $P_{n}$ to guarantee the convergence of $\sigma$ to zero. As long as this convergence is guaranteed, the designed performances can be realized.

\section{A. Sliding Surface}

According to Fig. 2, the sliding surface equation $\sigma=0$ is equivalent to

$$
\Lambda_{1} \widetilde{x_{R}}+\Lambda_{2} \widetilde{v_{G}}=0
$$

Substitute (3) and (5) into (7), we have

$$
\Lambda_{1}\left(x_{R}+n_{x}\right)+\Lambda_{2}\left(G_{v} v_{G}+n_{v}\right)=0
$$




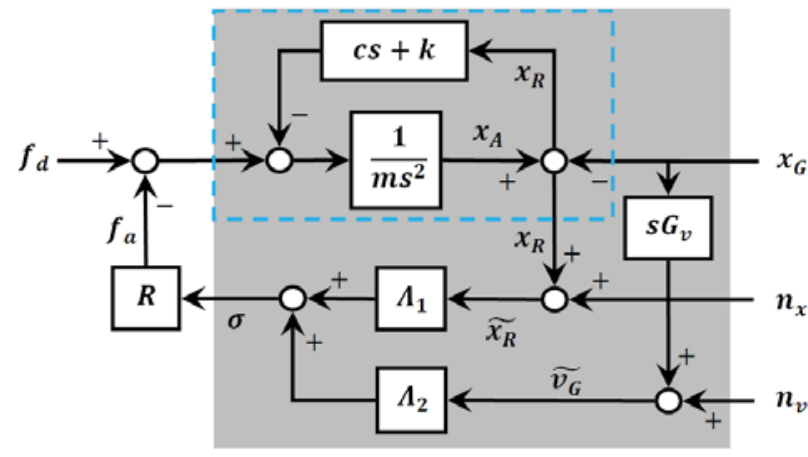

Fig. 2. FSSSC diagram with floor velocity measurement.

Apply the Laplace Transform, we have

$$
X_{A}=\frac{\Lambda_{1}-\Lambda_{2} s G_{v}}{\Lambda_{1}} X_{G}-N_{x}-\frac{\Lambda_{2}}{\Lambda_{1}} N_{v}
$$

where $X_{A}, X_{G}, N_{x}$, and $N_{v}$ are the Laplace transform of signals $x_{A}, x_{G}, n_{x}$, and $n_{v}$, respectively. It is equivalent to

$$
\frac{X_{A}}{X_{G}}=\mathbb{T}_{d}+\mathbb{R}_{d} \frac{N_{x}}{X_{G}}+\mathbb{S}_{d} \frac{N_{v}}{X_{G}},
$$

where $\mathbb{T}_{d}, \mathbb{R}_{d}$, and $\mathbb{S}_{d}$ are the designed transmissibility, the designed displacement-sensor-noise sensitivity, and the designed velocity-sensor-noise sensitivity, respectively. They are defined as

$$
\mathbb{T}_{d}=\frac{\Lambda_{1}-\Lambda_{2} S G_{v}}{\Lambda_{1}}, \mathbb{R}_{d}=-1, \mathbb{S}_{d}=\frac{-\Lambda_{2}}{\Lambda_{1}}
$$

The upper bound of the designed transmissibility, $\left|\overline{\mathbb{T}_{d}}\right|$, is derived as

$$
\left|\mathbb{T}_{d}\right| \leqslant\left|\overline{\mathbb{T}_{d}}\right|=\left|\mathbb{T}_{d}\right|+\left|\mathbb{R}_{d}\right|\left|\frac{N_{x}}{X_{G}}\right|+\left|\mathbb{S}_{d}\right|\left|\frac{N_{v}}{X_{G}}\right|,
$$

To make $\mathbb{T}_{d}$ more robust against the sensor noise, its upper bound $\left|\overline{\mathbb{T}_{d}}\right|$ has to be reduced. Since $\mathbb{R}_{d}=-1,\left|\overline{\mathbb{T}_{d}}\right|$ would approach to a limit $\left|\frac{N_{x}}{X_{G}}\right|$ at high frequencies. To reduce the upper bound at low frequencies by control, $\left|\mathbb{S}_{d}\right|$ has to be reduced. According to (11), $\mathbb{S}_{d}$ and $\mathbb{T}_{d}$ are related by

$$
\mathbb{T}_{d}-s G_{v} \mathbb{S}_{d}=1 \text {. }
$$

Therefore, to simultaneously improve both $\mathbb{S}_{d}$ and $\mathbb{T}_{d}$ is impossible with predefined geophone dynamics, which is of the same condition with the payload velocity measurement [7]. The sliding surface design has to make a trade-off between $\mathbb{S}_{d}$ and $\mathbb{T}_{d}$.

\section{B. Close-Loop Performance}

All the designed performances, $\mathbb{T}_{d}, \mathbb{R}_{d}$, and $\mathbb{S}_{d}$, can be realized as long as $\sigma=0$, which is the objective of the regulation. The regulator may be linear or nonlinear. If nonlinear regulator is applied and the convergence of $\sigma$ to zero is guaranteed, the designed transmissibility can be approximately realized. But there would be no closed-loop compliance. The switching control and the sliding mode control are possible candidates for the nonlinear regulator. If linear regulator is applied, the closed-loop performances can be theoretically calculated. In the rest of this subsection, the closed-loop performances are investigated assuming that linear converging control is applied.

Assuming that $R$ in Fig. 2 is linear, both closed-loop compliance $\mathbb{C}_{c}$ and transmissibility $\mathbb{T}_{c}$ can be calculated.

$$
\mathbb{C}_{c}=\frac{\frac{1}{R}}{\frac{1}{P R}+\frac{c s+k}{R}+\Lambda_{1}}, \mathbb{T}_{c}=\frac{\Lambda_{1}-\Lambda_{2} s G_{v}+\frac{c s+k}{R}}{\frac{1}{P R}+\frac{c s+k}{R}+\Lambda_{1}} .
$$

The closed-loop displacement-sensor-noise $\mathbb{R}_{c}$ and the closed-loop velocity-sensor-noise sensitivity $\mathbb{S}_{c}$ are calculated as

$$
\mathbb{R}_{c}=\frac{-\Lambda_{1}}{\frac{1}{P R}+\frac{c s+k}{R}+\Lambda_{1}}, \mathbb{S}_{c}=\frac{-\Lambda_{2}}{\frac{1}{P R}+\frac{c s+k}{R}+\Lambda_{1}}
$$

The upper bound of $\left|\mathbb{T}_{c}\right|$ is derived as

$$
\left|\mathbb{T}_{c}\right| \leqslant\left|\overline{\mathbb{T}_{c}}\right|=\left|\mathbb{T}_{c}\right|+\left|\mathbb{R}_{c}\right|\left|\frac{N_{x}}{X_{G}}\right|+\left|\mathbb{S}_{c}\right|\left|\frac{N_{v}}{X_{G}}\right| .
$$

If the open loop gain is so high that the approximations

$$
\begin{gathered}
\Lambda_{1}-\Lambda_{2} s G_{v}+\frac{c s+k}{R} \approx \Lambda_{1}-\Lambda_{2} s G_{v}, \\
\frac{1}{P R}+\frac{c s+k}{R}+\Lambda_{1} \approx \Lambda_{1},
\end{gathered}
$$

are feasible, we have $\mathbb{T}_{c}=\mathbb{T}_{d}, \mathbb{R}_{c}=\mathbb{R}_{d}$, and $\mathbb{S}_{c}=\mathbb{S}_{d}$. Also, the upper bound in (16) is exactly the same as (12) and $\left|\mathbb{C}_{c}\right|$ is reduced. The bottle neck to increase the regulator gain would be the actuator capacity, the time delay, and the flexible modes. Therefore, $R$ has to be properly designed to make the approximation (17) feasible at interested frequencies. As a result, design of $\mathbb{T}_{c}$ and $\mathbb{S}_{c}$ can be accomplished by the sliding surface design only.

\section{FSSSC With FLOOR ACCELERATION}

The FSSSC approach with floor acceleration measurement is illustrated in Fig. 3. The sliding surface is shaped by $\Lambda_{1}$ and $\Lambda_{3}$. The corresponding sliding surface design and the closed-loop performance are studied in this section.

\section{A. Sliding Surface}

According to Fig. 3, the sliding surface equation $\sigma=0$ is equivalent to

$$
\Lambda_{1} \widetilde{x_{R}}+\Lambda_{3} \widetilde{a_{G}}=0
$$

Substitute (3) and (6) into (18), we have

$$
\Lambda_{1}\left(x_{R}+n_{x}\right)+\Lambda_{3}\left(a_{G}+n_{a}\right)=0 .
$$

Apply the Laplace Transform, we have

$$
X_{A}=\frac{\Lambda_{1}-\Lambda_{3} s^{2}}{\Lambda_{1}} X_{G}-N_{x}-\frac{\Lambda_{3}}{\Lambda_{1}} N_{a}
$$

where $X_{A}, X_{G}, N_{x}$, and $N_{a}$ are the Laplace transform of signals $x_{A}, x_{G}, n_{x}$, and $n_{a}$, respectively. It is equivalent to

$$
\frac{X_{A}}{X_{G}}=\mathbb{T}_{d}+\mathbb{R}_{d} \frac{N_{x}}{X_{G}}+\mathbb{S}_{d} \frac{N_{a}}{X_{G}}
$$

where $\mathbb{T}_{d}, \mathbb{R}_{d}$, and $\mathbb{S}_{d}$ are the designed transmissibility, the designed displacement-sensor-noise sensitivity, and the designed acceleration-sensor-noise sensitivity, respectively. They are defined as

$$
\mathbb{T}_{d}=\frac{\Lambda_{1}-\Lambda_{3} s^{2}}{\Lambda_{1}}, \mathbb{R}_{d}=-1, \mathbb{S}_{d}=\frac{-\Lambda_{3}}{\Lambda_{1}}
$$




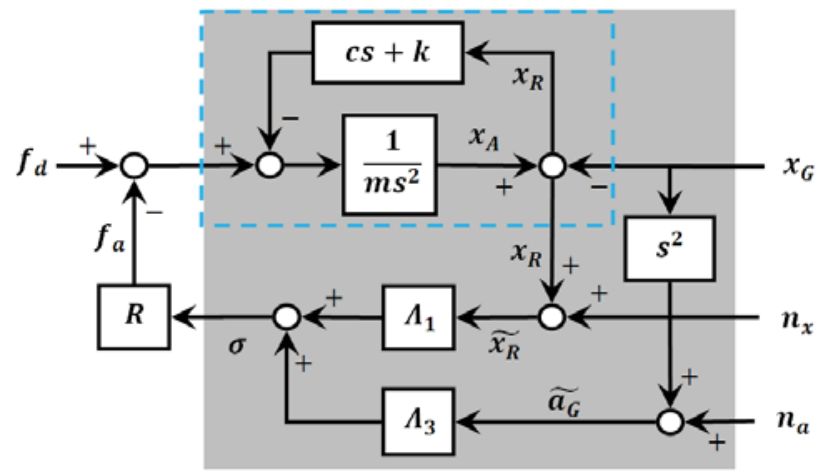

Fig. 3. FSSSC diagram with floor acceleration measurement.

The upper bound of the designed transmissibility, $\left|\overline{\mathbb{T}_{d}}\right|$, is derived as

$$
\left|\mathbb{T}_{d}\right| \leqslant\left|\overline{\mathbb{T}_{d}}\right|=\left|\mathbb{T}_{d}\right|+\left|\mathbb{R}_{d}\right|\left|\frac{N_{x}}{X_{G}}\right|+\left|\mathbb{S}_{d}\right|\left|\frac{N_{a}}{X_{G}}\right|
$$

To make $\mathbb{T}_{d}$ more robust against the sensor noise, its upper bound $\left|\overline{\mathbb{T}_{d}}\right|$ has to be reduced. Since $\mathbb{R}_{d}=-1,\left|\overline{\mathbb{T}_{d}}\right|$ would approach to a limit $\left|\frac{N_{x}}{X_{G}}\right|$ at high frequencies. To reduce the upper bound at low frequencies by control, $\left|\mathbb{S}_{d}\right|$ has to be reduced. According to (22), $\mathbb{S}_{d}$ and $\mathbb{T}_{d}$ are related by

$$
\mathbb{T}_{d}-s^{2} \mathbb{S}_{d}=1
$$

Therefore, to simultaneously improve both $\mathbb{S}_{d}$ and $\mathbb{T}_{d}$ is impossible. This scenario is exactly the same as the case of floor velocity feedback. The sliding surface design has to make a trade-off between $\mathbb{S}_{d}$ and $\mathbb{T}_{d}$.

\section{B. Close-Loop Performance}

The objective of the regulation is still $\sigma=0$ which is the condition to realize all the designed performances, $\mathbb{T}_{d}$, $\mathbb{R}_{d}$, and $\mathbb{S}_{d}$. The regulator design is similar to the case of floor velocity feedback. In this subsection, the closed-loop performances are investigated assuming that linear regulator is applied.

Assuming that $R$ in Fig. 3 is linear, both closed-loop compliance $\mathbb{C}_{c}$ and transmissibility $\mathbb{T}_{c}$ can be calculated.

$$
\mathbb{C}_{c}=\frac{\frac{1}{R}}{\frac{1}{P R}+\frac{c s+k}{R}+\Lambda_{1}}, \mathbb{T}_{c}=\frac{\Lambda_{1}-\Lambda_{3} s^{2}+\frac{c s+k}{R}}{\frac{1}{P R}+\frac{c s+k}{R}+\Lambda_{1}} .
$$

The closed-loop displacement-sensor-noise $\mathbb{R}_{c}$ and the closed-loop velocity-sensor-noise sensitivity $\mathbb{S}_{c}$ are calculated as

$$
\mathbb{R}_{c}=\frac{-\Lambda_{1}}{\frac{1}{P R}+\frac{c s+k}{R}+\Lambda_{1}}, \mathbb{S}_{c}=\frac{-\Lambda_{3}}{\frac{1}{P R}+\frac{c s+k}{R}+\Lambda_{1}}
$$

The upper bound of $\left|\mathbb{T}_{c}\right|$ is derived as

$$
\left|\mathbb{T}_{c}\right| \leqslant\left|\overline{\mathbb{T}_{c}}\right|=\left|\mathbb{T}_{c}\right|+\left|\mathbb{R}_{c}\right|\left|\frac{N_{x}}{X_{G}}\right|+\left|\mathbb{S}_{c}\right|\left|\frac{N_{v}}{X_{G}}\right| \text {. }
$$

If the open loop gain is so high that the approximations

$$
\begin{gathered}
\Lambda_{1}-\Lambda_{3} s^{2}+\frac{c s+k}{R} \approx \Lambda_{1}-\Lambda_{3} s^{2}, \\
\frac{1}{P R}+\frac{c s+k}{R}+\Lambda_{1} \approx \Lambda_{1},
\end{gathered}
$$

are feasible, we have $\mathbb{T}_{c}=\mathbb{T}_{d}, \mathbb{R}_{c}=\mathbb{R}_{d}$, and $\mathbb{S}_{c}=\mathbb{S}_{d}$. Also, the upper bound in (27) is exactly the same as (23) and $\left|\mathbb{C}_{c}\right|$ is reduced. The bottle neck to increase the regulator gain would be the actuator capacity, the time delay, and the flexible modes. Therefore, $R$ has to be properly designed to make the approximation (28) feasible at interested frequencies. As a result, design of $\mathbb{T}_{c}$ and $\mathbb{S}_{c}$ can be accomplished by the sliding surface design only.

\section{Example Sliding Surface Design}

\section{A. Sliding Surface with Floor Velocity}

The numerators and denominators of $\Lambda_{i}, \forall i \in\{1,2\}$ are denoted by $N_{i}$ and $D_{i}$, respectively. Let $D_{1}=\left(s^{2}+2 \omega_{\nu} \xi_{v} s+\right.$ $\left.\omega_{v}^{2}\right) D_{2}, \mathbb{T}_{d}$ and $\mathbb{S}_{d}$ have the following form according to (11).

$$
\mathbb{T}_{d}=\frac{N_{1}-N_{2} s^{3}}{N_{1}}, \quad \mathbb{S}_{d}=\frac{-N_{2}\left(s^{2}+2 \omega_{v} \xi_{v} s+\omega_{v}^{2}\right)}{N_{1}} .
$$

To achieve $\mathbb{S}_{d}(0)=0$, the constant term of the polynomial $N_{2}$ should be zero. Let $N_{2}=N_{2}^{\prime} s$, (29) becomes

$$
\mathbb{T}_{d}=\frac{N_{1}-N_{2}^{\prime} s^{4}}{N_{1}}, \quad \mathbb{S}_{d}=\frac{-N_{2}^{\prime} s\left(s^{2}+2 \omega_{v} \xi_{v} s+\omega_{v}^{2}\right)}{N_{1}} .
$$

$\mathbb{T}_{d}$ can be designed by the choice of $N_{1}$ and $N_{2}^{\prime}$. To achieve the $-40 \mathrm{~dB} / \mathrm{dec}$ decreasing rate of $\left|\mathbb{T}_{d}\right|$ at high frequencies, the denominator order should be the numerator order plus two. The lowest numerator order is three as the higher order can be canceled out by proper design of $N_{2}$. The lowest order of $\mathbb{T}_{d}$ is therefore five. If $\mathbb{T}_{d}$ is $5^{\text {th }}$-order, the two numerators can be designed as $N_{1}=a_{5} s^{5}+a_{4} s^{4}+a_{3} s^{3}+a_{2} s^{2}+a_{1} s+a_{0}$ and $N_{2}=a_{5} s^{2}+a_{4} s$ so that $\mathbb{T}_{d}$ has the form of

$$
\mathbb{T}_{d}=\frac{a_{3} s^{3}+a_{2} s^{2}+a_{1} s+a_{0}}{a_{5} s^{5}+a_{4} s^{4}+a_{3} s^{3}+a_{2} s^{2}+a_{1} s+a_{0}} .
$$

The five poles of $\mathbb{T}_{d}$ can be selected based on the criterions of stability and low resonant frequency. Subsequently, the five constants $a_{i}, \forall i \in\{1,2,3,4,5\}$ are uniquely determined. Based on (29), $\mathbb{S}_{d}$ has the form of

$$
\mathbb{S}_{d}=-\frac{\left(a_{5} s^{2}+a_{4} s\right)\left(s^{2}+2 \omega_{v} \xi_{v} s+\omega_{v}^{2}\right)}{a_{5} s^{5}+a_{4} s^{4}+a_{3} s^{3}+a_{2} s^{2}+a_{1} s+a_{0}} .
$$

Therefore, both $\mathbb{T}_{d}$ and $\mathbb{S}_{d}$ fulfill the design criterions. The design of $D_{1}$ and $D_{2}$ is to make $\Lambda_{1}$ and $\Lambda_{2}$ stable and to simplify the regulator design. If the order of $\mathbb{T}_{d}$ is increased, higher decreasing rate of $\left|\mathbb{T}_{d}\right|$ or lower $\left|\mathbb{S}_{d}\right|$ can be achieved. The trade-off would be the increased order of the controller.

\section{B. Sliding Surface with Floor Acceleration}

The numerators and denominators of $\Lambda_{i}, \forall i \in\{1,3\}$ are denoted by $N_{i}$ and $D_{i}$, respectively. Let $D_{1}=D_{2}$, (22) becomes

$$
\mathbb{T}_{d}=\frac{N_{1}-N_{3} s^{2}}{N_{1}}, \quad \mathbb{S}_{d}=-\frac{N_{3}}{N_{1}}
$$

To achieve $\mathbb{S}_{d}(0)=0$, the constant term of the polynomial $N_{3}$ should be zero. Let $N_{3}=N_{3}^{\prime} s$, (33) becomes

$$
\mathbb{T}_{d}=\frac{N_{1}-N_{3}^{\prime} s^{3}}{N_{1}}, \quad \mathbb{S}_{d}=-\frac{N_{3}^{\prime} s}{N_{1}} .
$$

$\mathbb{T}_{d}$ can be designed by the choice of $N_{1}$ and $N_{3}$. To achieve the $-40 \mathrm{~dB} / \mathrm{dec}$ decreasing rate of $\left|\mathbb{T}_{d}\right|$ at high frequencies, the 
denominator order should be the numerator order plus two. The lowest numerator order is two as the higher order can be canceled out by proper design of $N_{3}$. The lowest order of $\mathbb{T}_{d}$ is therefore four. If $\mathbb{T}_{d}$ is $4^{\text {th }}$-order, the two numerators can be designed as $N_{1}=a_{4} s^{4}+a_{3} s^{3}+a_{2} s^{2}+a_{1} s+a_{0}$ and $N_{2}=a_{4} s^{2}+a_{3} s$ so that $\mathbb{T}_{d}$ has the form of

$$
\mathbb{T}_{d}=\frac{a_{2} s^{2}+a_{1} s+a_{0}}{a_{4} s^{4}+a_{3} s^{3}+a_{2} s^{2}+a_{1} s+a_{0}} .
$$

The four poles of $\mathbb{T}_{d}$ can be selected based on the criterions of stability and low resonant frequency. Subsequently, the four constants $a_{i}, \forall i \in\{1,2,3,4\}$ are uniquely determined. Based on (33), $\mathbb{S}_{d}$ has the form of

$$
\mathbb{S}_{d}=-\frac{a_{4} s^{2}+a_{3} s}{a_{4} s^{4}+a_{3} s^{3}+a_{2} s^{2}+a_{1} s+a_{0}} .
$$

Therefore, both $\mathbb{T}_{d}$ and $\mathbb{S}_{d}$ fulfill the design criterions. The design of $D_{1}$ and $D_{2}$ is to make $\Lambda_{1}$ and $\Lambda_{2}$ stable and to simplify the regulator design. If the order of $\mathbb{T}_{d}$ is increased, higher decreasing rate of $\left|\mathbb{T}_{d}\right|$ or lower $\left|\mathbb{S}_{d}\right|$ can be achieved. The trade-off would be the increased order of the controller.

\section{Performance And Robustness}

If the regulator is linear, the closed-loop transmissibility and its upper bound can be theoretically calculated. The parameters of the geophone dynamics are assumed to be $\omega_{g}=2 \pi \mathrm{rad} / \mathrm{s}(1 \mathrm{~Hz})$ and $\xi_{g}=0.7$ so that the geophone dynamics is calculated as

$$
G_{v}=\frac{s^{2}}{s^{2}+8.796 s+39.48}
$$

We also assume that $\left|\frac{N_{v}(\omega)}{X_{G}(\omega)}\right|=0.2$ and $\left|\frac{N_{a}(\omega)}{X_{G}(\omega)}\right|=0.2, \forall \omega \geqslant 0$. $\left|\frac{N_{x}(\omega)}{X_{G}(\omega)}\right|, \forall \omega \geqslant 0$ takes a set of numbers $\{0.0001,0.001,0.01\}$ to show the influence of the displacement sensor noise. The example plant with different parameters will be used to test the performance robustness. The model parameters for a pneumatic plant are assumed to be $m=500 \mathrm{~kg}, c=894$ $\mathrm{N} \cdot \mathrm{s} / \mathrm{m}$, and $k=10^{4} \mathrm{~N} / \mathrm{m}$. The model parameters for an electromagnetic plant are assumed to be $m=500 \mathrm{~kg}, c=0$, and $k=-10^{4} \mathrm{~N} / \mathrm{m}$.

\section{A. Floor Velocity Feedback}

The five poles of the designed transmissibility are chosen as $[-1.4+1.4 i,-1.4-1.4 i,-1.4+1.4 i,-1.4-1.4 i,-2]$. The designed sliding surface is

$$
\Lambda_{1}=\frac{s^{2}+2.8 s+3.92}{s^{2}+8.796 s+39.48}, \Lambda_{2}=\frac{s^{2}+7.6 s}{s^{3}+4.8 s^{2}+9.52 s+7.84} \text {. }
$$

The designed transmissibility is

$$
\mathbb{T}_{d}=\frac{26.88(s+0.9333)\left(s^{2}+1.05 s+1.225\right)}{(s+2)\left(s^{2}+2.8 s+3.92\right)^{2}} .
$$

$R$ is designed as a PID controller.

$$
R=\frac{2 \times 10^{5}\left(s^{2}+2.8 s+3.92\right)}{s} .
$$

The designed transmissibility $\mathbb{T}_{d}$ and the closed-loop transmissibility $\mathbb{T}_{c}$ are compared in Fig. 4 for both pneumatic and electromagnetic plants. For different plants, both $\mathbb{T}_{d}$ and $\mathbb{T}_{c}$

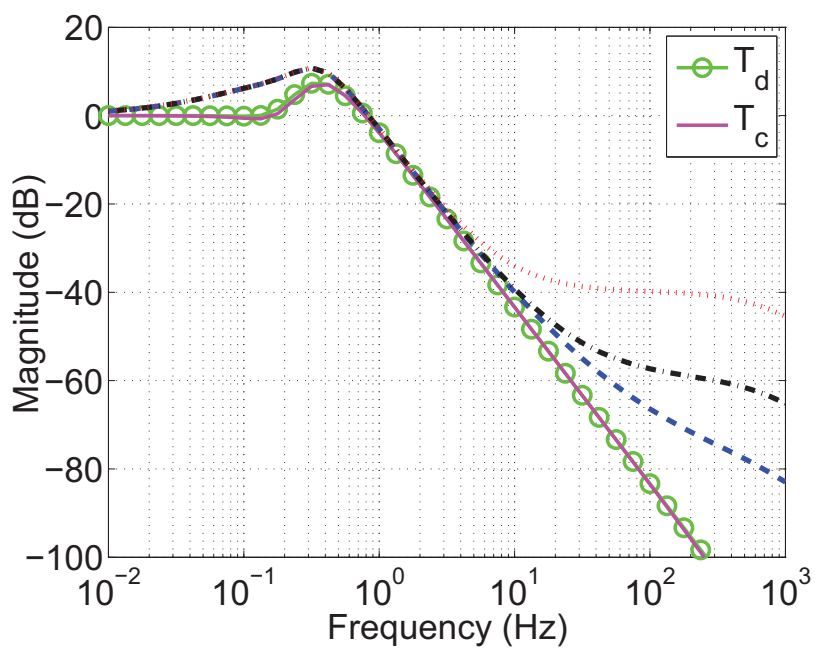

(a) For the pneumatic plant.

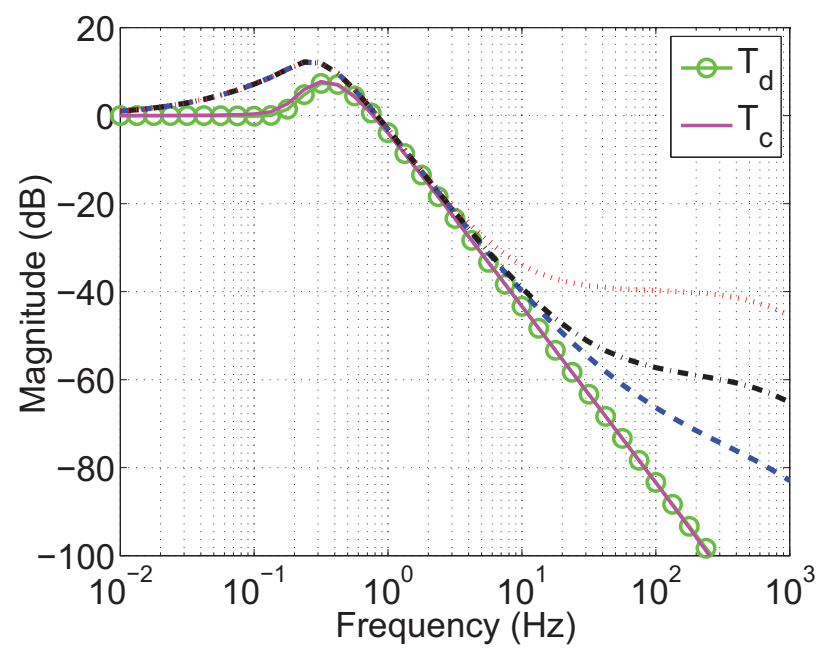

(b) For the electromagnetic plant.

Fig. 4. $\quad\left|\mathbb{T}_{c}\right|$ and $\left|\mathbb{T}_{d}\right|$ with floor absolute velocity measurement. $\mathbb{T}_{d}$ is designed to be $5^{\text {th }}$ order. The blue dashed line, the black dashed-dotted line, and the red dotted line are $\left|\overline{\mathbb{T}_{c}}\right|$ calculated for the ratio of $\left|\frac{N_{x}(\omega)}{X_{G}(\omega)}\right|, \forall \omega \geqslant 0$ is $0.0001,0.001$, and 0.01 , respectively.

are almost identical, which indicates that the transmissibility is robust against the plant uncertainties. For each plant, $\mathbb{T}_{c}$ and $\mathbb{T}_{d}$ coincide and they both have the resonant peak at around $0.3 \mathrm{~Hz}$. The high frequency decreasing rate is maintained as $-40 \mathrm{~dB} / \mathrm{dec}$. However, $\mathbb{T}_{c}$ is close to its upper bound only at low frequencies. The $\left|\overline{\mathbb{T}_{c}}\right|$ converges to the values of $\left|\frac{N_{x}(\omega)}{X_{G}(\omega)}\right|$ at high frequencies. It indicates that robustness of the realized transmissibility is highly dependent on the displacement sensor noises at high frequencies.

\section{B. Floor Acceleration Feedback}

The four poles of the designed transmissibility are chosen as $[-1.4+1.4 i,-1.4-1.4 i,-1.4+1.4 i,-1.4-1.4 i,-2]$. The designed sliding surface is

$$
\Lambda_{1}=1, \Lambda_{3}=\frac{s^{2}+5.6 s}{s^{4}+5.6 s^{3}+15.68 s^{2}+21.95 s+15.37} .
$$




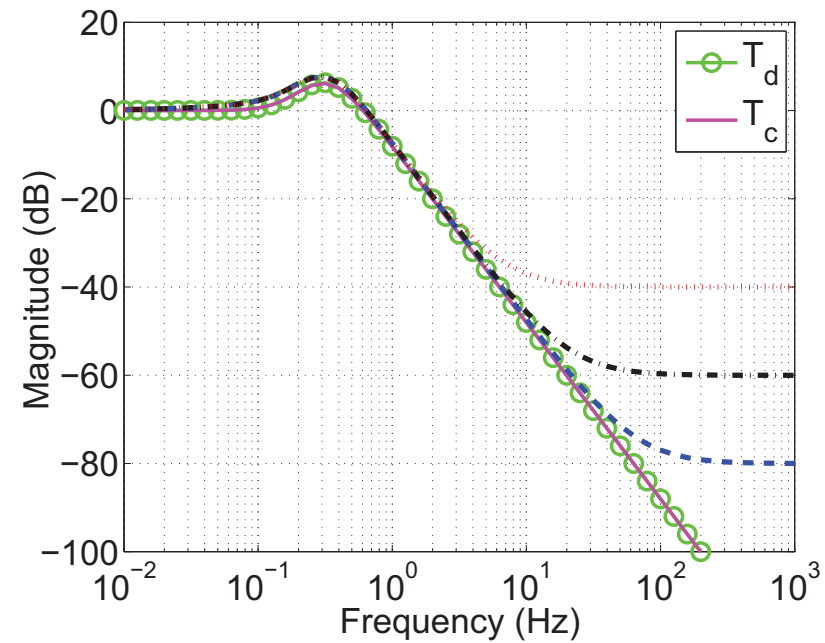

(a) For the pneumatic plant.

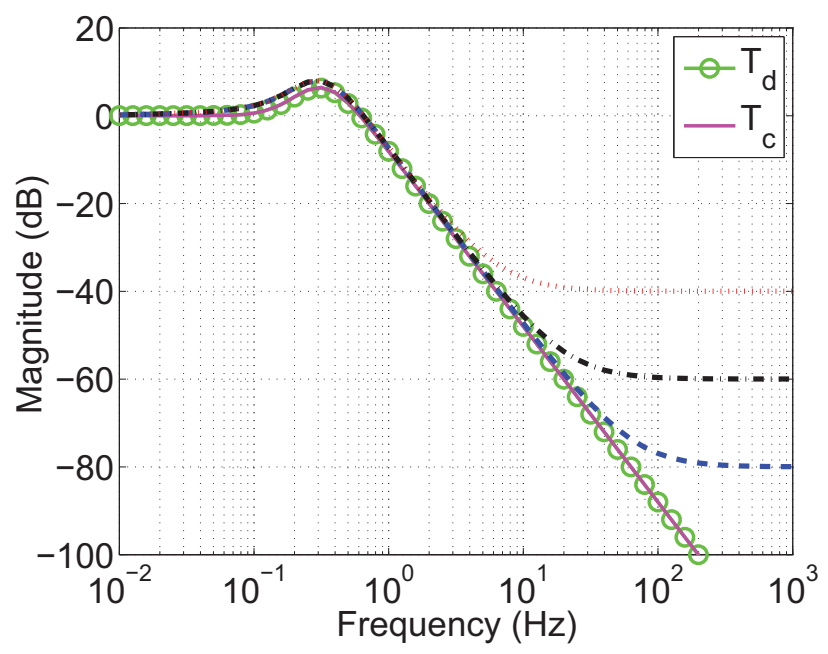

(b) For the electromagnetic plant.

Fig. 5. $\left|\mathbb{T}_{c}\right|$ and $\left|\mathbb{T}_{d}\right|$ with floor absolute acceleration measurement. $\mathbb{T}_{d}$ is designed to be $4^{\text {th }}$ order. The blue dashed line, the black dashed-dotted line, and the red dotted line are $\left|\overline{\mathbb{T}_{c}}\right|$ calculated for the ratio of $\left|\frac{N_{x}(\omega)}{X_{G}(\omega)}\right|, \forall \omega \geqslant 0$ is $0.0001,0.001$, and 0.01 , respectively.

The designed transmissibility is

$$
\mathbb{T}_{d}=\frac{15.68\left(s^{2}+1.4 s+0.98\right)}{\left(s^{2}+2.8 s+3.92\right)^{2}}
$$

$R$ is designed as the same PID controller.

$$
R=\frac{2 \times 10^{5}\left(s^{2}+2.8 s+3.92\right)}{s} .
$$

The designed transmissibility $\mathbb{T}_{d}$ and the closed-loop transmissibility $\mathbb{T}_{c}$ are compared in Fig. 5 for both pneumatic and electromagnetic plants. For different plants, both $\mathbb{T}_{d}$ and $\mathbb{T}_{c}$ are almost identical, which indicates that the transmissibility is robust against the plant uncertainties. For each plant, $\mathbb{T}_{c}$ and $\mathbb{T}_{d}$ coincide and they both have the resonant peak at around $0.3 \mathrm{~Hz}$. The high frequency decreasing rate is maintained as $-40 \mathrm{~dB} / \mathrm{dec}$. However, $\mathbb{T}_{c}$ is close to its upper bound only at low frequencies. The $\left|\overline{\mathbb{T}_{c}}\right|$ converges to the values of $\left|\frac{N_{x}(\omega)}{X_{G}(\omega)}\right|$ at high frequencies. It indicates that robustness of the realized transmissibility is highly dependent on the displacement sensor noises at high frequencies. Compared to the Fig. 4, the transmissibility at low frequencies is more robust.

\section{CONCLUSION}

This paper studies 1-DOF AVIS control using FrequencyShaped Sliding Surface Control (FSSSC) approach based on measurement schemes of the relative displacement and the floor absolute velocity/acceleration. The sliding surface designs incorporating the sensor dynamics are provided. Performance robustness against the sensor noises is investigated. Theoretical calculations are provided for the FSSSC design based on a 1-DOF example plant. For both measurement schemes, the calculation results show that the designed performance can be realized by a high-gain regulator. The performance is robust at low frequencies. The performance robustness at high frequencies strongly depends on the displacement sensor noise.

\section{ACKNOWLEDGMENTS}

This work is a part of the Dutch IOP-EMVT program and is supported financially by SenterNovem, an agency of the Dutch Ministry of Economic Affairs.

\section{REFERENCES}

[1] D. Karnopp, M.J. Crosby, and R.A. Harwood, "Vibration Control Using Semi-Active Force Generators", ASME Journal of Engineering for Industry, vol. 96, pp. 619-626, 1974.

[2] Katsuhide WATANABE, Shinji HARA, Yoichi KANEMITSU, Takahide HAGA, Kenichi YANO, Takayuki MIZUNO, and Ryuta KATAMURA, "Combination of $H_{\infty}$ and PI Control for an Electromagnetically Levitated Vibration Isolation System", Proc. 35th Conference on Decision and Control, Kobe, Japan, Dec. 1996.

[3] C. Ding, A.A.H. Damen, P.P.J. van den Bosch, and J.L.G. Janssen, "Vibration Isolation of an Electromagnetic Actuator with Passive Gravity Compensation", Proc. 2nd International Conference on Computer and Automation Engineering, Singapore, Feb. 2010.

[4] L. Zuo and J.J.E. Slotine, "Robust vibration isolation via frequencyshaped sliding control and modal decomposition", Journal of Sound and Vibration, vol. 285, no. 4-5, pp. 1123-1149, Aug. 2005.

[5] C. Ding, A.A.H. Damen, P.P.J. van den Bosch, "Stabilization and Vibration Isolation of a Contactless Electromagnetic Isolator: A FrequencyShaped Sliding Surface Control Approach", Proc. 2010 IEEE/ASME International Conference on Advanced Intelligent Mechatronics, Montreal, Canada, Jul. 2010.

[6] M. Heertjes, K. de Graaff, and J.G. van der Toorn, "Active Vibration Isolation of Metrology Frames; A Modal Decoupled Control Design", Journal of Sound and Acoustics-Transactions of the ASME, vol. 127, issue 3, pp. 223-233, Jun. 2005.

[7] C. Ding, A.A.H. Damen, P.P.J. van den Bosch, "Robust Vibration Isolation Control by Frequency-Shaped Sliding Surface Control with Geophone Dynamics", To be presented on 36th Annual Conference of the IEEE Industrial Electronics Society, The Renaissance Glendale Hotel and Spa, Greater Phoenix, Arizona, USA, Nov. 2010.

[8] Jinpyo Honga and Kyihwan Park, "Design and control of six degreeof-freedom active vibration isolation table", Review of Scientific Instruments, vol 81, issue 3, 2010. 